\title{
Successful removal of an intrapontine haematoma
}

\author{
T. D O C Z I A N D D. G . T . T H O M A S \\ From the Gough Cooper Department of Neurosurgery, Institute of Neurology, \\ The National Hospital, London
}

SUMMARY A patient with progressive signs and symptoms suggestive of a pontine lesion is described. Intracranial investigations, CAT scan, vertebral angiography, and lumbar pneumoencephalogram revealed a space-occupying lesion of the pons and midbrain. The exact nature of the lesion was not established before the operation. A posterior fossa exploration was performed and a pontine haematoma was discovered and evacuated. The pathological specimen was designated as a cryptic arteriovenous malformation. Preoperative neurological deficits disappeared except for minimal left sixth nerve palsy and mild truncal ataxia.

Dandy (1969) was the first to report the evacuation of a haematoma of the brainstem. Since that time a few additional reports have been made but the surgical treatment of this condition is still an extreme rarity. Recently interest has been focused on the feasibility of a more aggressive surgical approach to brainstem glioma as patients with cystic brainstem tumours can be long-term survivors after aspiration and radiation therapy as reported by Lassiter et al. (1971). The present paper is a report of the clinical and pathological findings in a patient who presented with a clinical picture of brainstem tumour and had successful evacuation of a pontine and midbrain haematoma.

\section{Case report}

This 14 year old girl had fallen into a swimming pool about three months before her admission to hospital. There appeared to be no definite head injury. Subsequently she had very serious headache in the back of the neck and head. There was no loss of consciousness and no vomiting. The headaches persisted intermittently and after about one month she started to complain of paraesthesia affecting the right side of the face, arm, and leg. She had no weakness. She continued at school. Two weeks before her admission she developed left sided ptosis associated with double vision but painless. At the time of admission she was conscious, normally orientated but rather drowsy.

Address for reprint requests: D. G. T. Thomas, The Gough Cooper Department of Neurosurgery, Institute of Neurology, The National Hospital, Queen Square, London WC1N 3BG.

Accepted 14 May 1979
She was normotensive and general physical examination did not reveal any abnormal findings. The optic discs showed no swelling. There was a slight left sided ptosis with a mild left sixth nerve palsy causing diplopia. Tone was slightly increased in all limbs, with brisk tendon reflexes. She had an equivocal left plantar response. Power and sensation in the limbs appeared normal to objective examination although there was occasional paraesthesia in the right limbs. She had very severe, mainly truncal, ataxia, and was not able to stand or walk. Intracranial investigation with a CAT scan revealed an extensive high attenuation area in the brainstem, involving predominantly the posterior part, crossing the midline, but more extensive on the left. The brainstem was swollen, compressing the fourth ventricle, and the high attenuation area extended from the lower pons into the midbrain with swelling of the left cerebral peduncle, encroaching on the interpeduncular cisterns. There was low CAT attenuation with some mass effect seen in the region of the left hypothalamus and temporal region. There was no change after contrast injection. There was no hydrocephalus (Fig. 1). A vertebral angiogram confirmed the presence of swelling on the left side of the pons with forward displacement of the basilar artery and slight backward displacement of the choroidal point. No pathological vessels were seen. A lumbar pneumoencephalogram confirmed that there was an intrinsic swelling of the brainstem, pons, and midbrain extending up to the cerebral peduncle on the left side (Fig. 2a, b). The density of the lesion shown on the CAT scan was compatible with a haematoma but the other 


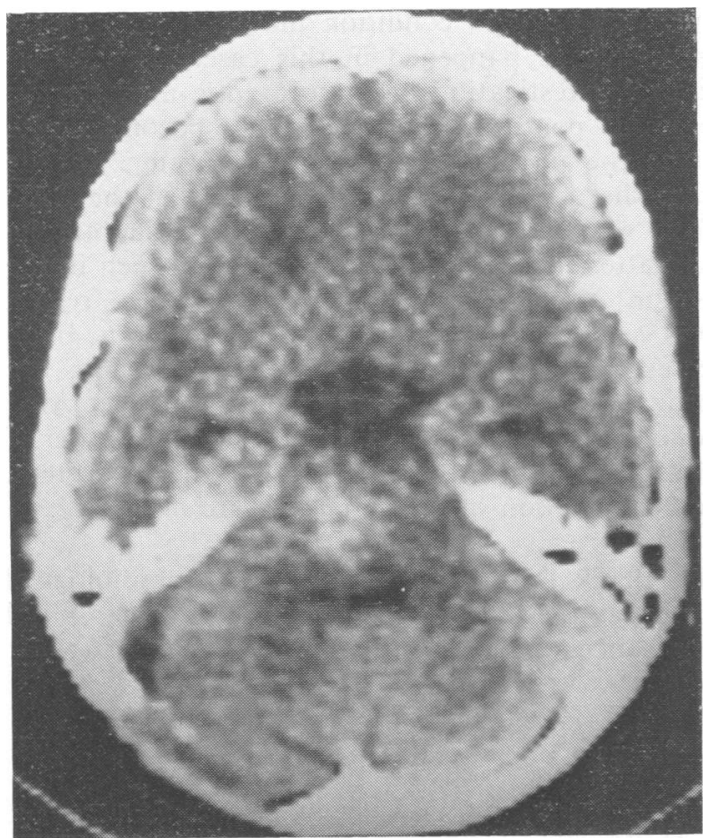

Fig. 1 Computerised axial tomography after administration of contrast medium shows extensive high attenuation in the brainstem, predominantly involving the posterior part, crossing the midline but more extensive on the left. The brainstem is swollen compressing the fourth ventricle.

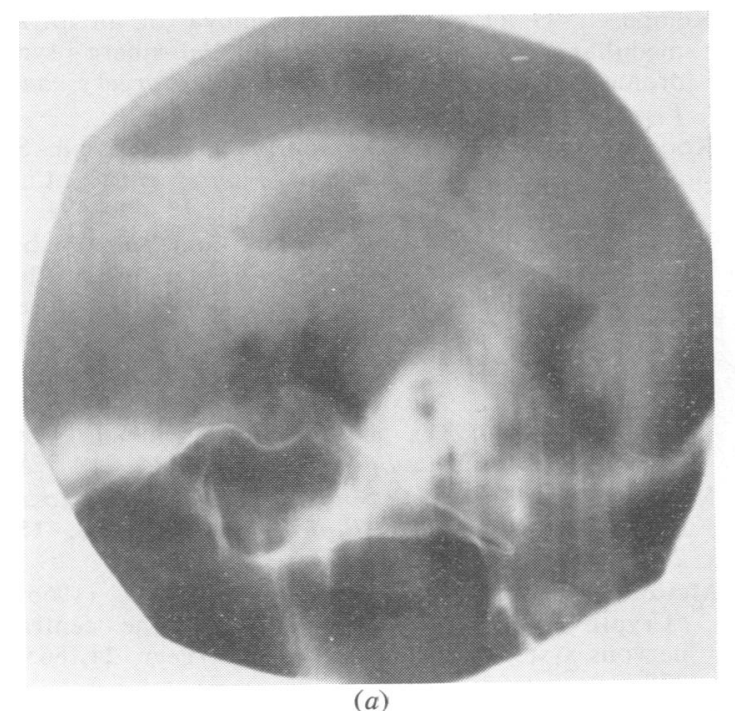

features of the scan were very suggestive of an underlying tumour. After the pneumoencephalogram a posterior fossa exploration was performed. The left cerebellopontine angle, the free margin of the tentorium and the lateral aspect of the pons, and the brainstem were visualised. The pons was extremely swollen and a brown discoloured area was seen in its lateral aspect at the level of the tentorium. On needling the brainstem a haematoma was located. A small opening was made in the lateral aspect of the pons at the level of the tentorium on the left and approximately $5 \mathrm{ml}$ of dark brown fluid was removed. There was no sign of angioma or tumour. After the haematoma was evacuated the brainstem returned to normal size. The patient made a good postoperative recovery with rapid improvement in the drowsiness and ataxia. The diplopia has also lessened gradually. $A$ repeat CAT scan showed virtual resolution of the haematoma and the low attenuation in the left side of the pons, the midbrain, and the thalamus had disappeared. There was no suggestion of tumour. The pathological specimen was reported as clotted blood.

\section{Discussion}

Brainstem haematomas can be defined as subependymal or intra-axial. The subependymal haematoma has the typical appearance of an intraventricular expanding lesion, and in most cases

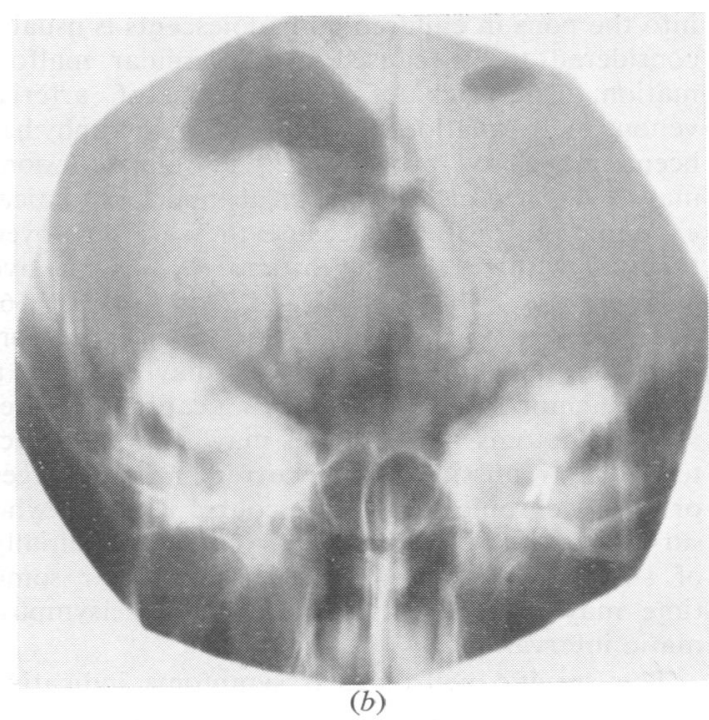

Fig. 2 Pneumoencephalogram shows expansion of the upper part of the pons and the midbrain extending up to the cerebral peduncle on the left side. The fourth ventricle is only slightly displaced. (a) Lateral and (b) $A-P$ jiew. (A-P view reversed to match CAT scan). 
the haemorrhagic collection from the peripheral portion of the tegmentum pontis tends to spread towards the lumen of the fourth ventricle being limited only by the ependymal layer. In some cases this is disrupted giving rise to subarachnoid haemorrhage. Intra-axial haematomas are not distinguishable from intrinsic brainstem expanding tumours, which are generally not considered suitable for surgical therapy. Gros et al. (1948), Scoville and Poppen (1949), Kempe (1964), Matson (1968), Dandy (1969), Obrador et al. (1970) Abroms et al. (1971) Kowada et al. (1971) and Papo et al (1976) reported successful evacuation of subependymal brainstem haematomas. Myers et al. (1961) Kowada et al. (1971), and Scott et al. (1973) reported successful evacuation of intraaxial brainstem haematoma. Brainstem haematoma may present as subarachnoid haemorrhage accompanied by brainstem stroke in an acute clinical picture as reported by Koos et al. (1969) and Murphy (1972). In other cases brainstem intrinsic tumour accompanied by raised intracranial pressure caused by cerebrospinal fluid pathway occlusion was the initial diagnosis (Gros et al., 1948; Myers et al., 1961; Dandy, 1969; Obrador et al, 1970; Scott et al., 1973).

Kempe (1964) described a brainstem haematoma presenting with Wallenberg's syndrome. Abroms et al. (1971) described a case in which multiple sclerosis was established at the first stage and later successful evacuation of a pontine haematoma was performed. Spontaneous haemorrhage into the pons in children and adolescents is usually considered to be caused by a vascular malformation. The lack of visualisation of arterial venous malformation on vertebral angiography has been emphasised previously. These small lesions may even escape pathological and anatomical examination, probably because they are destroyed together with the brain substance by any massive haemorrhage. McCormick and Nofzinger (1966) have referred to them as cryptic vascular malformations. This type of angioma may produce a fatal haemorrhage without having caused any previous symptoms. The diseases may also be characterised by remissions and recurrent haemorrhages or take a chronic progressive course. Patients who survive pontine haemorrhages complain initially of sudden onset of headache which after some time may disappear to recur after an asymptomatic interval.

The specific neurological symptoms indicative of the pontine site of the process may vary in severity. The patient may either show temporary improvement with subsequent aggravation of the symptoms or the condition may deteriorate progressively as happened in this case. Neuroradiological investigation of these cryptic angiomas in cases of pontine haemorrhage has become easier since the introduction of CAT scanning. In our case the density of the lesion shown on the CAT scan was compatible with a haematoma but the low attenuation with some mass effect seen in the region of the hypothalmus and temporal region was very suggestive of an underlying tumour. In all cases when the patients' neurological deficits are progressive and the nature of the space-occupying lesion in the brainstem is doubtful, exploration of the posterior fossa, inspection of the brainstem, and evacuation of a haematoma are necessary.

We thank Dr I. Mosely for the neuroradiological investigations and Miss R. E. Green for secretarial assistance.

\section{References}

Abroms, I. F., Yessayan, L., Shillito, L., and Barlow, C. F. (1971). Spontaneous intracerebral haemorrhage in patient suspected of multiple sclerosis. Journal of Neurology, Neurosurgery, and Psychiatry, 34, 157-162.

Dandy, W. E. (1969). The Brain, pp. 642-643. Harper and Row: New York.

Gros, C., Giraud, G., and Latour, H. (1948). Hématome spontané de la calotte protubérantielle opéré et gueri. Presse Médicale, 56, 890-894.

Kempe, L. G. (1964). Surgical removal of an intramedullary haematoma simulating. Wallenberg's syndrome. Journal of Neurology, Neurosurgery, and Psychiatry, 27, 78-80.

Koos, W. T., Sunder-Plassmann, M., and Salah, S. (1969). Successful removal of a large intrapontine hematoma. Journal of Neurosurgery, 13, 690-694.

Kowada, M., Ito, Z., Matsuoka, Sh., and Yamaguchi, K. (1971). Primary pontine haemorrhage revealed by pneumonencephalo-roulette tomography and a report on surgically treated cases. Acta Neurochirurgica, 25, 269-276.

Lassiter, K. R. L., Alexandra, E., Davis, C. H., and Kelly, D. L. (1971). Surgical treatment of brain stem gliomas. Journal of Neurosurgery, 34, 719-725.

Matson, D. D. (1968). Surgery of posterior fossa tumors in childhood. Clinical Neurosurgery, 15, 247-264.

McCormick, W. F., and Nofzinger, J. D. (1966). "Cryptic" vascular malformation of the central nervous system. Journal of Neurosurgery, 24, 865875.

Murphy, M. G. (1972). Successful evacuation of acute pontine hematoma. Journal of Neurosurgery, 37, 224-225. 
Myers, J., Scott, M., and Silverstein, A. (1961). Cystic hemangioblastoma of pons. Journal of Neurosurgery, 18, 694-697.

Obrador, S., Dierssen, G., and Odoriz, B. J. (1970). Surgical evacuation of a pontine-medullary hematoma. Journal of Neurosurgery, 33, 82-84.

Papo, I., Pasquini, U., and Salvolini, U. (1976). Subependymal brainstem hematomas: a report of 2 cases. Neuroradiology, 11, 279-282.

Scott, B. B., Seeger, J. F., and Schneider, R. C. (1973).
Successful evacuation of a pontine hematoma secondary to rupture of a pathologically diagnosed "cryptic" vascular malformation. Journal of Neurosurgery, 39, 104-108.

Scoville, W. B., and Poppen, J. L. (1949). Intrapeduncular hemorrhage of the brain: successful operative approach with evacuation of clot and a seven and one-fourth year observation period. Archives of Neurology and Psychiatry (Chicago), 61, 688-694. 